\title{
FSI methods for seismic analysis of sloshing tank problems (Corrigendum)
}

\author{
Zuhal Ozdemir, Mhamed Souli and Yasin M. Fahjan
}

Mécanique \& Industries 11, 133-147 (2010), DOI: 10.1051/meca/2010025

This corrigendum allows the authors to complete the acknowledgements of their article.

Acknowledgements. The authors would like to deeply thank Professor Sami A. Kilic and Bachelor of Science student Ceki Erginbas for the design of the experimental setup. The sloshing experiment was funded by Grant No. 07 HT102 of the Bogazici University's Research Fund (B.U. Arfon), for which Professor Kilic is the main investigator.

The authors would like also to express their deepest gratitude to Professor Erdal Safak of Kandilli Observatory of Bogazici University for careful review of the manuscript and his valuable recommendations. This work is supported by the Scientific and Technological Research Council of Turkey (TUBITAK-MAG) through contract number 108M607 and by Research Fund of Gebze Institute of Technology under project number 2009-A-25.

The first author is granted by Eiffel excellence scholarship of the French Ministry of Foreign and European Affairs. 